\title{
LA EMULSIÓN BITUMINOSA COMO ALTERNATIVA ECOLÓGICA EN LAS ESTABILIZACIONES DE SUELOS PARA FIRMES, EN VÍAS DE BAJA INTENSIDAD DE TRÁFICO
}

\author{
(THE BITUMINOUS EMULSION AS AN ECOLOGICAL ALTERNATIVE IN SOIL \\ STABILIZATIONS FOR ROADBEDS ON LOW TRAFFIC FLOW ROADS)
}

\author{
J.M. Martín-Montalvo y Vera, Ingeniero Agrónomo \\ TRAGSA \\ E. García-Vaquero Vaquero \\ Departamento de Construcción y Vías Rurales de la LPM
}

Fecha de recepción $27-$ IV - 93

$113-23$

ESPAÑA

\section{RESUMEN}

La realización de vías de comunicación ocasiona, con frecuencia, ciertos impactos ambientales debidos a:

1.- Los importantes movimientos de tierra que el logro del perfil adecuado exige.

2.- La extracción de materiales de graveras o canteras necesarias para realizar estabilizaciones granulométricas de terrenos de mala calidad.

En los caminos rurales el primer motivo señalado no alcanza una entidad significativa ya que, por razones de economía y por permitirlo las características del tráfico, son muy reducidos los volumenes de excavación de tierras.

No sucede lo mismo con el indicado en segundo lugar, puesto que al ser sustancial el volumen de obra que precisa este tipo de estabilización, se puede ocasionar una alteración ecológica de alguna importancia en las zonas de desarrollo de estas obras.

Este artículo intenta mostrar los medios disponibles en la actualidad, encaminados a reducir ese impacto, mediante el empleo de técnicas y materiales que no impliquen el uso de tierras de préstamo.

\section{SUMMARY}

Road construction often cause certain environmental impact, due to:

1.- The big earthmovings required to gain the ideal grade profile.

2.- The extraction of materials from gravels mine or quaries necessary to implement granular stabilizations of poor quality soils.

In rurals roads the first point have not significant entity, as for the saving cause and for the traffic characteristics, excavation quantities are very short.

It is not the case of the second point, as for the great amount of earthwork that this type of stabilization needs, can cause environmental changes of some importance in zones of developement of this works.

This paper attemp to show present available methods, designed to reduce impact, by means of the usage of technics and materials that needs not to operate with spoil earths.

\section{INTRODUCCIÓN}

Las vías denominadas de baja intensidad de tráfico suponen en los países desarrollados proporciones de hasta el setenta y cinco por ciento sobre el total de la longitud de sus redes viarias. Entas regiones actualmente en desarrollo, constituyen un elemento necesario para alcanzar el mismo. 
106

Informes de la Construcción. Vol. 45 n" 425-426, mayo/junio - julio/agosto 1993

En España, que no podía ser excepción respecto a esa tendencia, se ha producido un primer intento de normalización del proceso de dimensionamiento de firmes. Así, por la Orden de 12 de marzo de 1976 , por la que se aprueban las Instrucciones 6.1.IC 1975 y 6.2.IC 1975 de "firmes flexibles" y "firmes rígidos" (BOE 4-10-1976), se introduce el concepto de Categoría de Tráfico, y se empiezan a normalizar las secciones de firme para tráfico ligero, proyectándose y construyéndose esas secciones, no sólo en las nuevas carreteras estatales (con esos tipos de tráfico) sino en toda clase de vías de baja intensidad de tráfi$\mathrm{co}$.

Posteriormente, la Orden de 26 de marzo de 1980 por la que se aprueba la Instrucción 6.3.I.C, "refuerzo de firmes" (BOE 31-5-1980), supuso un aumento generalizado de los espesores de firmes flexibles. No obstante esa disposición, hoy es una realidad que cada Administración utiliza, con preferencia, su práctica y técnica de probada eficacia en el dimensionado de secciones de este tipo de caminos de baja intensidad de tráfico.

Es evidente que el desarrollo de las poblaciones rurales, la búsqueda de lo que se ha dado en denominar "ocio ecológico", el turismo rural, etc., aumentarán sin duda la presión sobre el medio, y demandarán en un futuro no lejano el desarrollo de la red viaria que hemos denominado de baja intensidad de tráfico.

En estas vías, que ya se vienen realizando desde los últimos años y que en el futuro se desarrollarán en mayor medida, los suelos para las explanadas deben ser, salvo excepciones, los que se encuentren en la misma traza por donde se proyecte el camino. Los costes totales permisibles en este tipo de infraestructuras no justifican transportes de suelos a ciertas distancias, lo que implica que se debe partir del máximo aprovechamiento de los materiales locales. Aunque en este tipo de vías se pueden llegar a admitir calidades de explanada inferiores a las exigibles en carreteras más importantes, cada día es más frecuente encontrar dificultades para disponer de materiales autóctonos (muchas veces marginales) que cumplan con unas condiciones mínimas para ser utilizados como estructura del firme.

En este artículo se pretende mostrar que existe una alternativa económica y técnicamente apropiada para utilizar estos materiales menos adecuados, empleando como adiciones diferentes tipos de emulsiones asfálticas para estabilizar los suelos mencionados, dentro de las limitaciones fijadas en el PG.
3 (Art. 511.2.1.3.). Asimismo, se mostrará que esas estabilizaciones mejoran en gran medida la capacidad portante de los firmes, al comportarse la emulsión bituminosa como ligante de los materiales granulares empleados y que dotan al camino de unas propiedades impermeabilizantes que inciden en la durabilidad del mismo y, por tanto, en su conservación.

Por otra parte, cabe recordar que la utilización de materiales autóctonos contribuye notablemente, no sólo a la economía en la construcción de dichas vías, sino también al mantenimiento de las condiciones ecológicas y medioambientales de la zona. A este respecto hay que señalar la cantidad de impactos ambientales que se registran como consecuencia de la explotación de canteras, areneros, graveras, etc., así como por la utilización de pedrizas y otros derrubios naturales aprovechados para la fabricación de áridos.

\section{CATEGORÍAS DE TRÁFICO Y SUELOS}

Antes de proseguir mostrando otro tipo de consideraciones respecto a las denominadas vías de baja intensidad de tráfico, lo primero que procede es intentar clasificar éstas en función de algunos criterios sencillos.

En primer lugar, y dado que cualquier método de dimensionamiento de firmes utiliza como variable fundamental, en el comportamiento de ellos, la influencia del tráfico, será este parámetro el empleado como primer criterio de clasificación. Si nos referimos al tráfico que soportan estas vías, la Instrucción 6.1 y 2 IC lo clasifica como de categoría T4; es decir, menos de 50 vehículos pesados por día y por sentido en la vía que se proyecte durante el año de puesta en servicio. Parece lógico partir de esta limitación impuesta para toda la categoría y utilizar ese mismo criterio de clasificación.

Así es posible realizar una clasificación del tráfico de las vías de baja densidad, en la que se establecen cinco categorías en función del número diario de vehículos pesados por sentido en el momento de la puesta en servicio (cuadro 1).

Como en muchas ocasiones no resulta sencillo estimar el número aproximado de vehículos pesados diarios que se espera circulen por la vía a proyectar, se recurre entonces a utilizar otras clasificaciones con un criterio subsidiario de la presentada anteriormente (cuadro 2). 
Otro criterio sencillo para ordenar las vías de baja intensidad de trafico, es el de considerar la capacidad soporte de la explanada como un factor fundamental en el comportamiento de un firme. Para clasificar los suelos en función de la capacidad soporte, se suele emplear como práctica habitual el valor del índice CBR, estableciendo cuatro categorías de calidad ascendente (cuadro 3)

Cuadro 1

\begin{tabular}{|c|c|}
\hline $\begin{array}{c}\text { CATEGORÍA DE } \\
\text { TRAFICO }\end{array}$ & $\begin{array}{c}\text { No DIARIO DE VEHÍCULOS } \\
\text { PESADOS POR SENTIDO EN } \\
\text { EL MOMENTO DE LA PUESTA } \\
\text { EN SERVICIO }\end{array}$ \\
\hline $\mathrm{T} 41$ & $25-49$ \\
\hline $\mathrm{T} 42$ & $12-24$ \\
\hline $\mathrm{T} 43$ & $6-11$ \\
\hline $\mathrm{T} 44$ & $2-5$ \\
\hline $\mathrm{T} 45$ & $0-1$ \\
\hline
\end{tabular}

Cuadro 2

\section{CATEGORÍAS DE TRÁFICO EN FUNCIÓN DEL TIPO Y} USO DE LA VÍA

\begin{tabular}{|c|c|c|c|}
\hline \multirow{4}{*}{$\begin{array}{l}\text { VÍAS } \\
\text { AGRÍCOLAS } \\
\text { Y } \\
\text { FORESTALES }\end{array}$} & \multirow{2}{*}{$\begin{array}{l}\text { AGRICULTURA } \\
\text { INTENSIVA }\end{array}$} & PRINCIPALES & $\mathrm{T} 43$ \\
\hline & & SECUNDARIAS & $\mathrm{T} 44$ \\
\hline & \multirow{2}{*}{$\begin{array}{l}\text { AGRICULTURA, } \\
\text { EXTENSIVA Y } \\
\text { VÍAS RURALES }\end{array}$} & PRINCIPALES & T44 \\
\hline & & SECUNDARIAS & T45 \\
\hline \multirow{4}{*}{$\begin{array}{l}\text { VÍAS } \\
\text { DE USO } \\
\text { GENERAL }\end{array}$} & \multirow{4}{*}{$\begin{array}{c}\mathrm{N}^{\circ} \text { DE } \\
\text { HABITANTES } \\
\text { DE LOS NÚCLEOS } \\
\text { DE POBLACIÓN A } \\
\text { LOS QUE SIRVE } \\
\text { LA VÍA }\end{array}$} & $<200$ & $\mathrm{~T} 44$ \\
\hline & & $200-500$ & T43 \\
\hline & & $500-2000$ & T42 \\
\hline & & $>2000$ & T41 \\
\hline
\end{tabular}

Cuadro 3

\begin{tabular}{|c|c|}
\hline $\begin{array}{c}\text { CATEGORÍA DEL } \\
\text { SUELO }\end{array}$ & ÍNDICE C.B.R. \\
\hline E 0 & $3-5$ \\
\hline E 1 & $6-10$ \\
\hline E 2 & $11-20$ \\
\hline E 3 & $>20$ \\
\hline
\end{tabular}

(c) Consejo Superior de Investigaciones Científicas Licencia Creative Commons 3.0 España (by-nc)

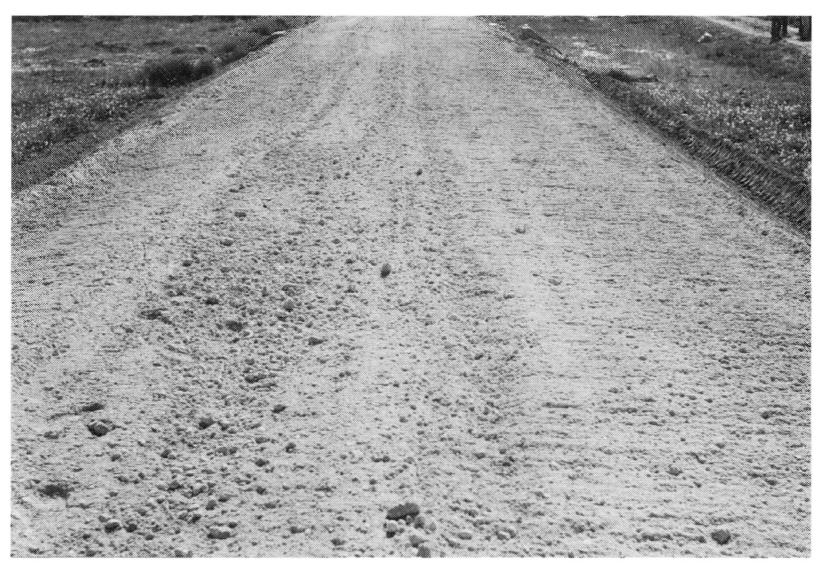

Fig. 1.- Zahorra natural extendida sobre caja previamente formada.
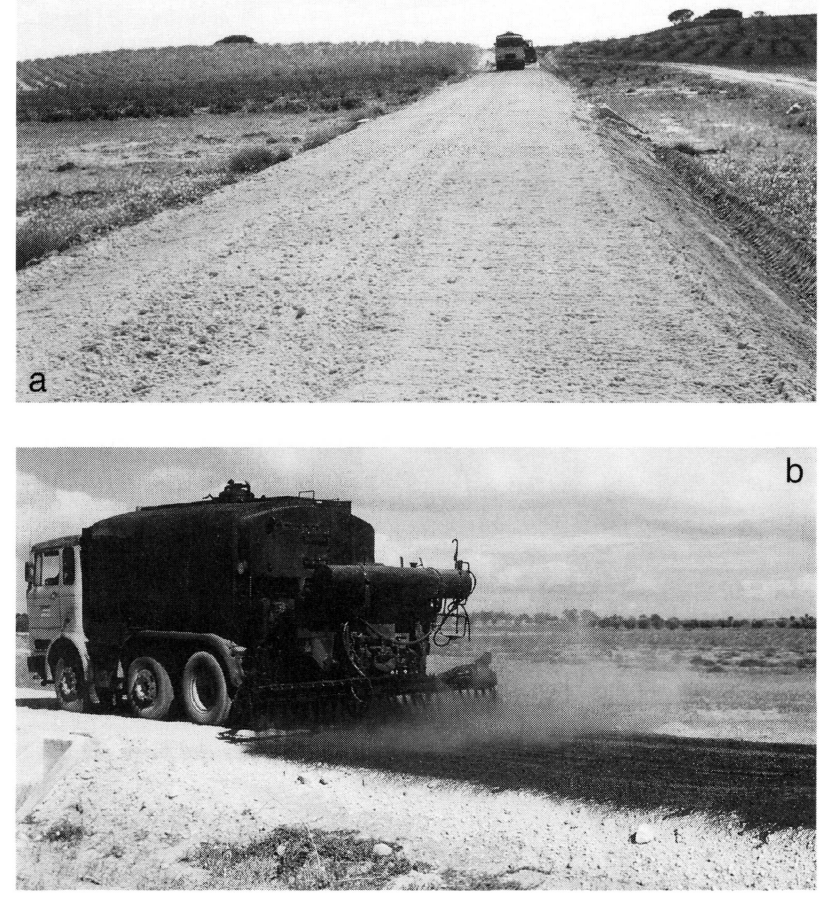

Fig. 2.- a) Primer riego con emulsión asfáltica. b) Primer riego con emulsión asfáltica. Detalle de cisterna con rampa.

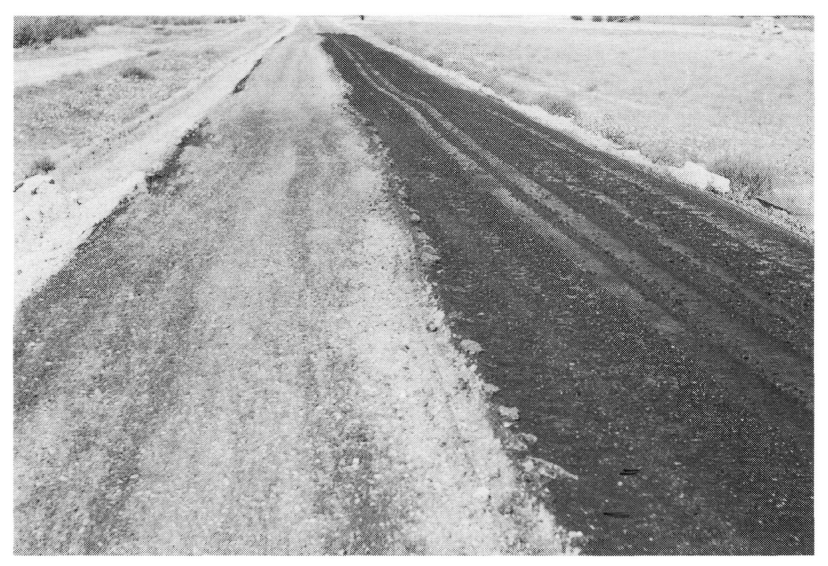

Fig. 3.- Aspecto del tratamiento después del pase de cisterna. 


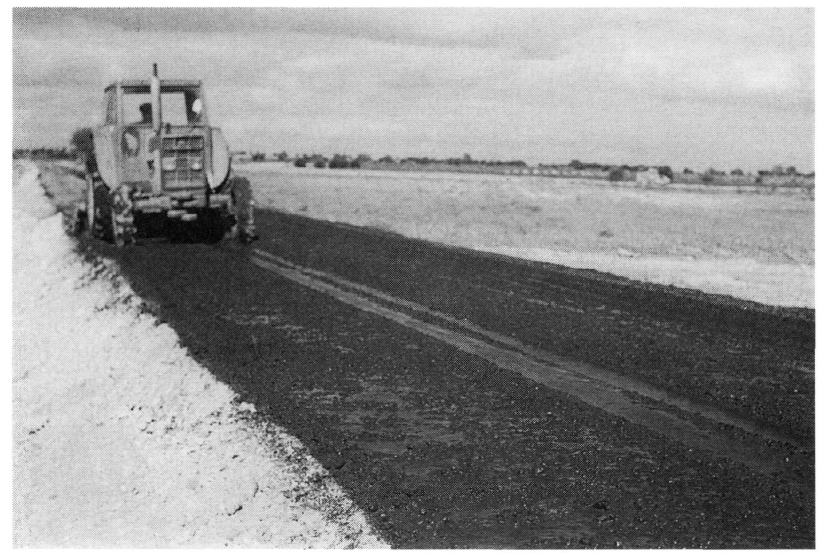

Fig. 4.- Primer pase de fresadora.

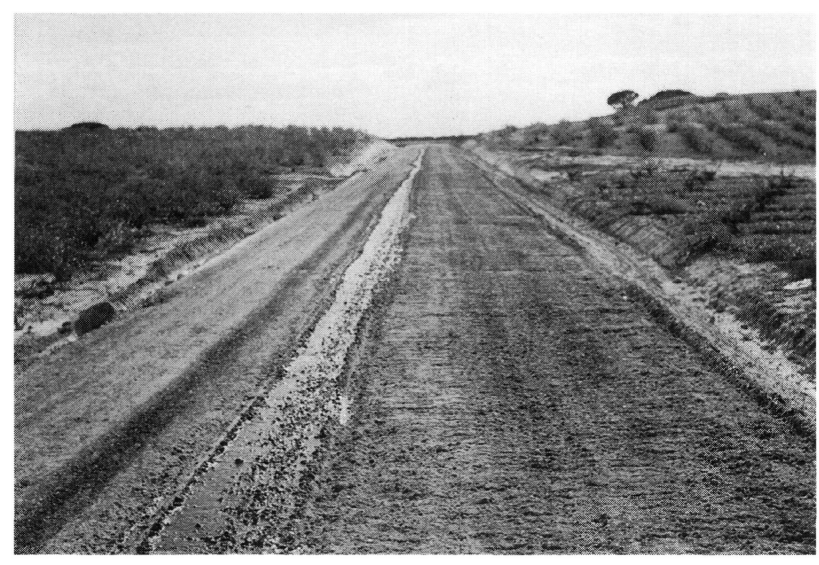

Fig. 4 bis .- Aspecto del tratamiento después del pase de fresadora.

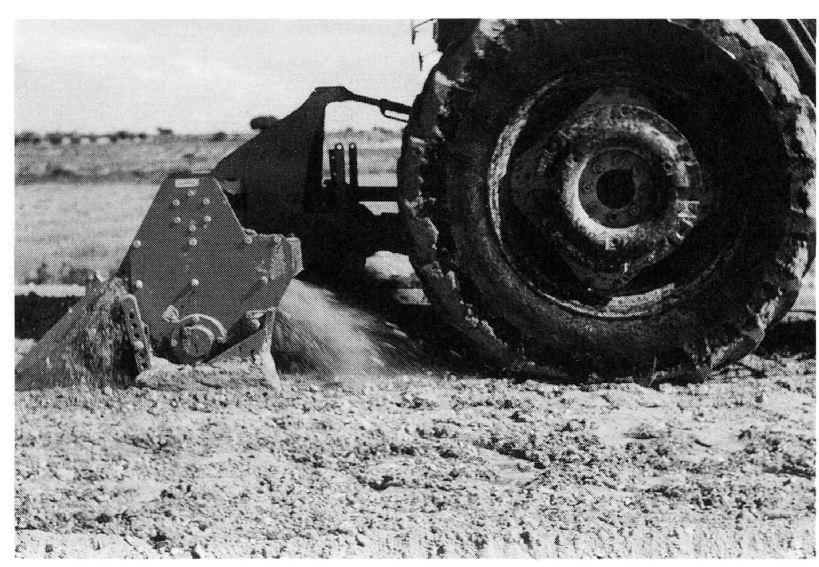

Fig. 5.- Primer pase de fresadora. Detalle de mezclado.

(c) Consejo Superior de Investigaciones Científicas Licencia Creative Commons 3.0 España (by-nc)

\section{ESTABILIZACIONES DE SUELOS}

Podría decirse, con carácter general, que cualquier capa constituyente de un firme está formada por materiales, o bien de una única naturaleza, o bien por la combinación de varios de diferente naturaleza: áridos naturales y adiciones. Las capas granulares están formadas, exclusivamente, por áridos naturales, por lo que, en este caso, se habla de suelos con estabilización granulométrica. En el resto, la estabilización de los suelos se efectúa a partir de un terreno y un aglomerante u otras adiciones.

Tradicionalmente en España se venían utilizando, en la construcción de la red viaria de baja intensidad de tráfico, capas de firmes granulares formadas por áridos de granulometría definida (zahorras), o capas formadas por áridos de granulometría limitada (macadam). Esta ultima, si bien es una técnica muy correcta, está en clara regresión debido a las dificultades de ejecución que plantea. El empleo de zahorras naturales en capas de base (y en ocasiones más que frecuentes, como capa de rodadura) se ha generalizado por varios motivos: primero, por su economía, si se dispone de los materiales a pie de obra o en zonas próximas (lo que evita el encarecimiento de la obra al no tener apenas transporte); segundo, por su facilidad de extracción, que permite paliar los impactos medioambientales al no ser muy costosos los trabajos de acondicionamiento o restauración de esos yacimientos granulares.

Sin embargo, actualmente es cada vez más frecuente el uso de materiales de los denominados zahorras artificiales o de machaqueo, al no ser fácil, a veces, encontrar materiales granulares naturales. En este caso, existe un evidente encarecimiento del coste.

Por ello, hoy en día se está generalizando el sistema de estabilización de los suelos existentes en la explanada del camino a proyectar. Los suelos estabilizados tienen unas características muy variables, en función de las que tengan los suelos de partida y del sistema empleado para lograr la estabilización.

Así pues, se podría hablar de dos tipos de estabilizaciones. En primer lugar, de estabilización mecánica cuando el proceso consiste en mezclar el suelo existente con otro de características diferentes que mejoran su calidad. Y en segundo lugar, de estabilización con adiciones, en las que $=$ se incorporan al suelo productos que actúan física y/o químicamente con el mismo, consiguiendo unas mejores características. En ambos casos, se pretende el máximo apro- 
vechamiento de los materiales locales. Sobre todo en vías de baja intensidad de tráfico, en que el transporte de materiales a unas ciertas distancias, incide de una manera elevadísima en el coste final del firme construido.

La estabilización mecánica se plantea cuando los suelos de la explanación no son de la calidad exigible y, a distancia reducida, se encuentran suelos de materiales complementarios. Como ya se ha comentado, no hay muchas zonas en las que existan materiales de la calidad adecuada. En estos casos habría que recurrir a utilizar materiales obtenidos mediante procesos de transformación más o menos complejos. Nos estamos refiriendo al empleo de materiales procedentes de canteras en explotación o al de plantas de machaqueo, tanto fijas como móviles, en las que se obtengan materiales granulares a partir de piedras calizas, cuarcitas, granitos en descomposición, formaciones de pedrizas, etc. No obstante, si no disponemos de canteras en las proximidades de la obra, estas otras explotaciones provisionales no son fáciles de montar por existir muchas dificultades para obtener los necesarios permisos de los organismos competentes, ante los problemas derivados de las medidas de protección del medio ambiente.

Por todo lo anterior, actualmente, en este tipo de vías es cada día más frecuente la estabilización de suelos mediante el empleo de aditivos. Dentro de los utilizables en la construcción de caminos se pueden considerar varios grupos principales de ellos:

CALES AÉREAS
CONGLOMERANTES HIDRÁULICOS
- Cementos.
- Cales hidráulicas.
CONGLOMERADOS PUZOLÁNICOS
- Escorias granuladas de alto horno
- Cenizas volantes silicoaluminosas.
- Puzolanas naturales.
LIGANTES HIDROCARBONADOS
- Alquitranes.
- Betunes fluidificados.
- Betunes fluxados.
- Betunes asfálticos.
- Emulsiones bituminosas.

(c) Consejo Superior de Investigaciones Científicas Licencia Creative Commons 3.0 España (by-nc)

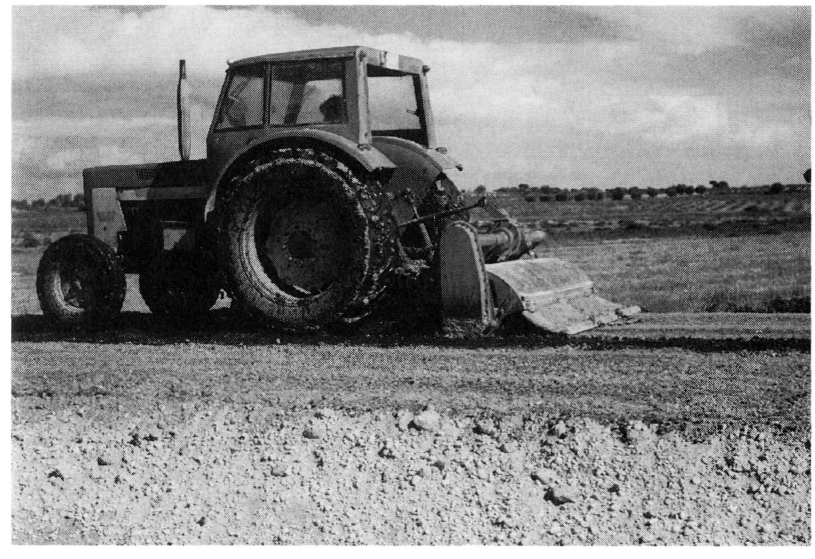

Fig. 6.- Detalle del segundo pase de fresadora. Detalle de mezclado.

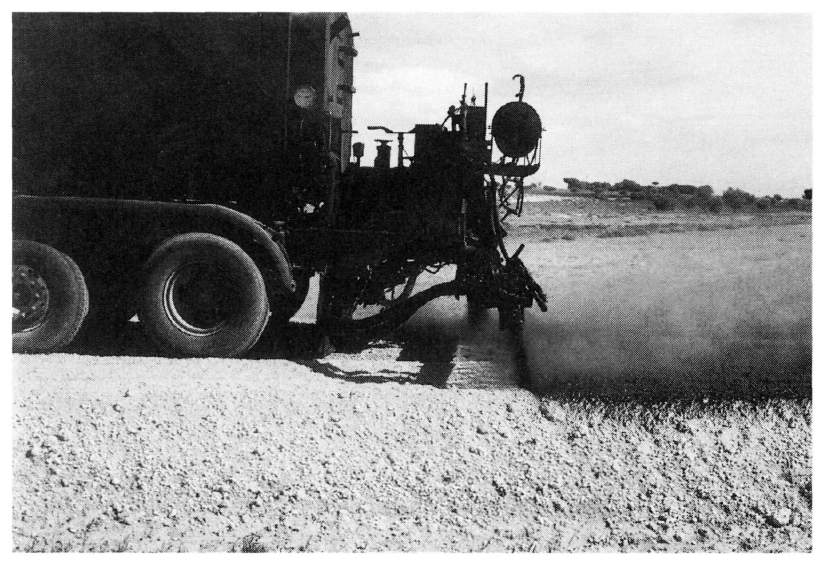

Fig. 7.- Segundo pase de cisterna de riego con emulsión.

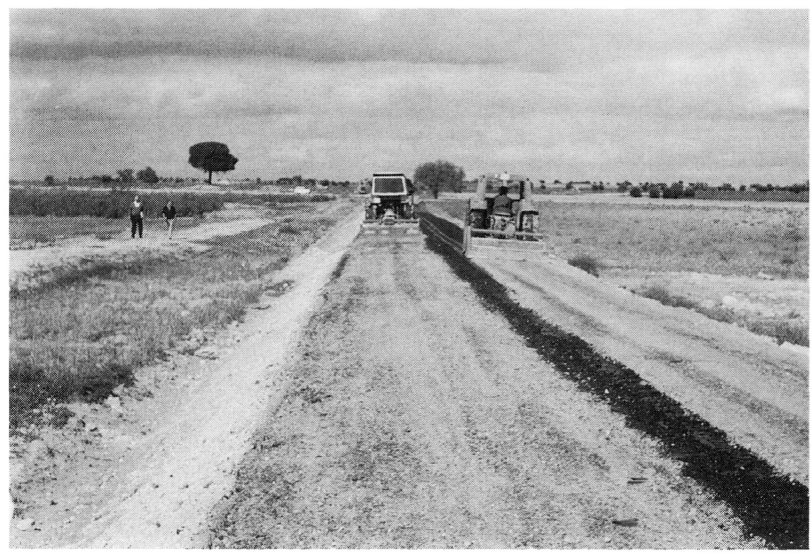

Fig. 8.- Detalle del segundo pase de fresadora. Detalle de mezclado. 


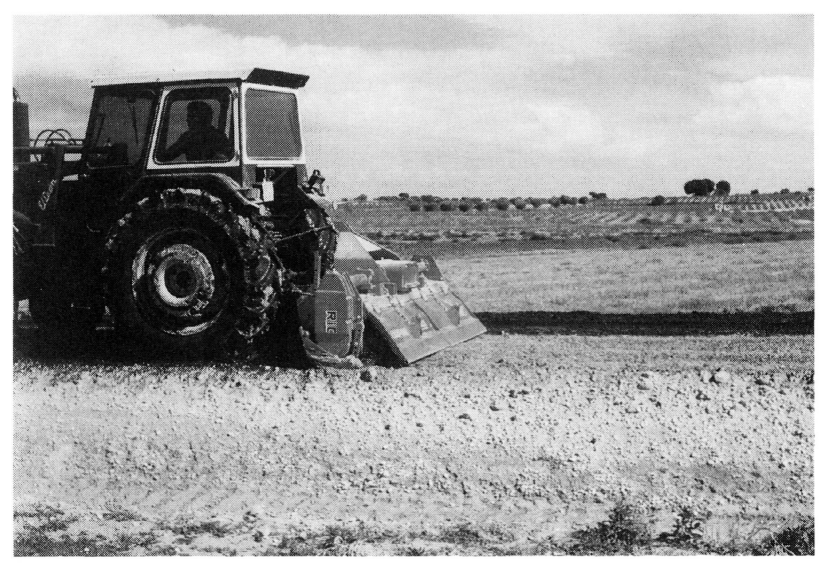

Fig. 9.- Detalle del segundo pase de fresadora. Detalle de mezclado.

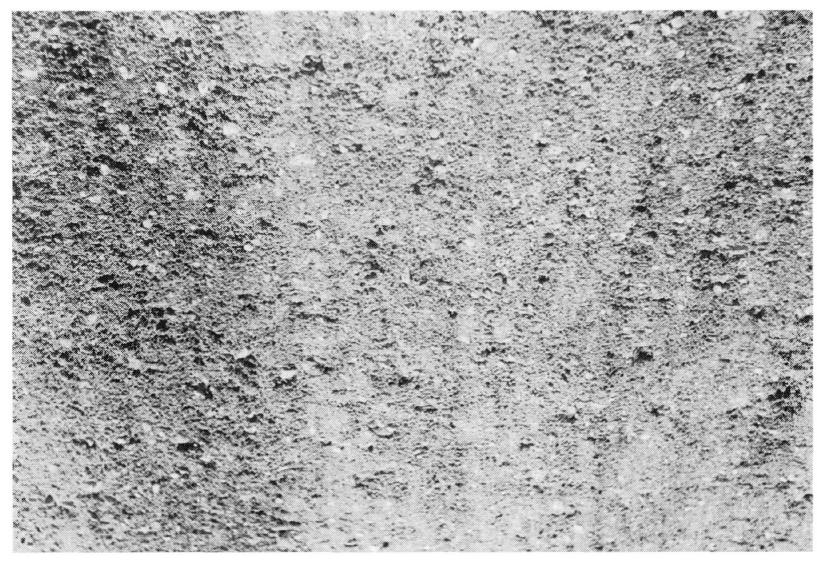

Fig. 10.- Aspecto del material regado y mezclado in situ.

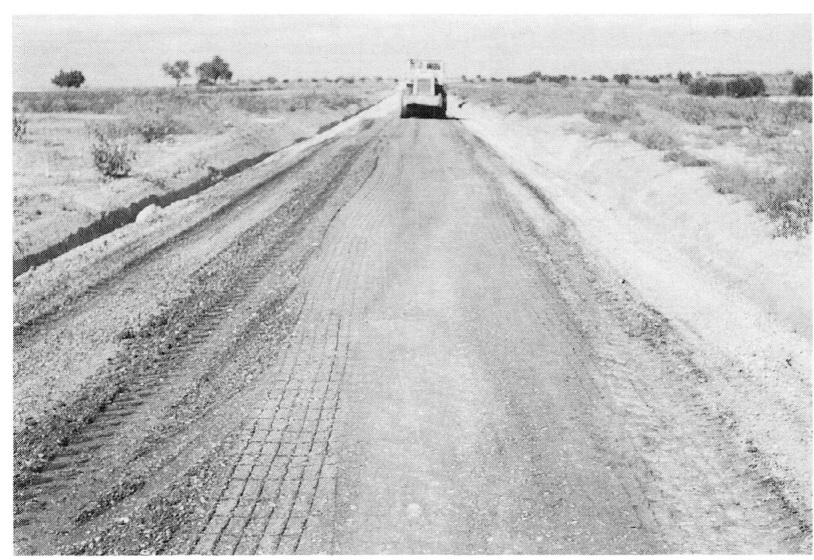

Fig. 11.- Compactación con vibro liso autopropulsado.
De todos los citados, hay que separar, por su escaso empleo en la construcción de firmes, las cales hidráulicas y las puzolanas naturales. Actualmente tampoco se utilizan en vialidad los alquitranes -por su rápido envejecimiento y alta toxicidad- y apenas los betunes fluidificados y fluxados -por el empleo de productos volátiles, como fluidificantes, de alto coste-.

Igualmente, habría que descartar los betunes asfálticos, que aunque se usan en construcción de firmes, no se deben emplear como aditivos en estabilizaciones, sino en la construcción de capas con mezclas bituminosas en caliente, dado su alto coste.

El resto, viene siendo utilizado habitualmente en estabilizaciones de suelos. Así, por ejemplo las cales aéreas se emplean para la estabilización in situ de suelos arcillosos con elevada plasticidad y/o elevada humedad natural. Los cementos se emplean para la estabilización in situ de suelos de reducida plasticidad. Las escorias granuladas de alto horno y las cenizas volantes silicoaluminosas, pueden ser utilizadas con ventaja como sustitutivos del cemento o en mezcla con él, siempre y cuando el camino a construir no esté lejos de los centros de producción de esos materiales, aunque es preciso mencionar las dificultades que presenta su distribución sobre el terreno.

Para clarificar ideas, es posible afirmar que en función de la categoría del suelo se pueden presentar unas soluciones de estabilizaciones in situ que combinan calidad de firme y bajo coste del mismo (cuadro 4)

Cuadro 4

\begin{tabular}{|c|c|}
\hline $\begin{array}{l}\text { CATEGORÍA DEL } \\
\text { SUELO }\end{array}$ & $\begin{array}{c}\text { ADITIVO MÁS APROPIADO } \\
\text { PARA ESTABILIZAR A MÁS } \\
\text { BA.JO COSTE }\end{array}$ \\
\hline E 0 & CAL AÉREA (1) \\
\hline E 1 & $\begin{array}{l}\text { CAL ÁREA/CEMENTO } \\
\text { (Y/O SUSTITUTIVOS) }\end{array}$ \\
\hline E 2 & $\begin{array}{l}\text { CEMENTO (Y/O } \\
\text { SUSTITUTIVOS)/ } \\
\text { EMULSIÓN BITUMINOSA }\end{array}$ \\
\hline E 3 & $\begin{array}{l}\text { EMULSIÓN BITUMINOSA } \\
\text { (2) }=\end{array}$ \\
\hline
\end{tabular}

(1) Sólo para mejorar explanaciones y sübbases.

(2) Siempre que no se trate de suelos confinados. 


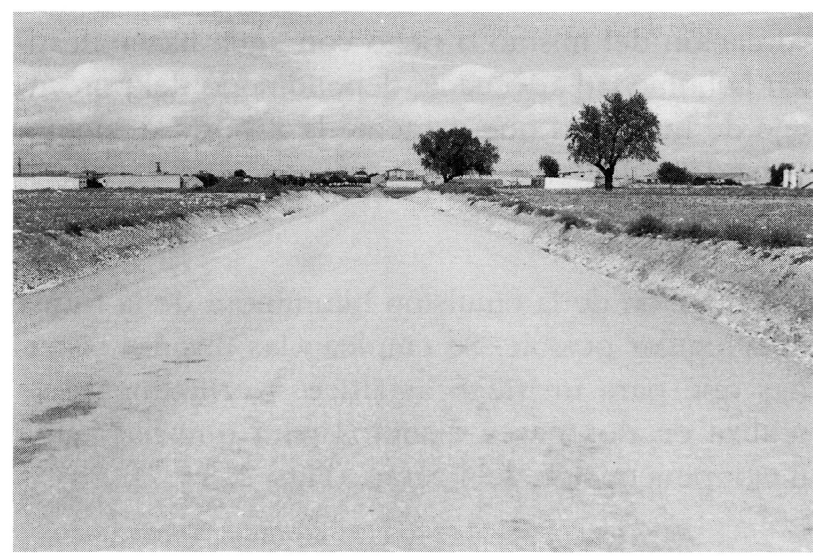

Fig. 12.- Aspecto final del camino después de compactación con neumáticos.

Se comprende que cuanto más se avanza hacia suelos de mejor categoría (tipo E3), mayor calidad presentará el firme (aunque puede tener un coste más alto) ya que las soluciones apuntadas para el suelo de categoría máxima, son utilizables en los de categorías inferiores, mejorando notablemente la calidad de ese firme (o capa de firme) aunque, eso sí, con un mayor coste.

Con todo lo anterior, puede decirse que en las vías de baja intensidad de tráfico — vías que requieren bajas inversiones de construcción - y para capas de base y/o sub-base, la estabilización in situ de los firmes constituye una de las mejores opciones. Por lo visto anteriormente, lógicamente una de las soluciones de más calidad la constituirá el empleo de emulsión bituminosa.

También es necesario dejar bien claro que si el camino a proyectar tiene una intensidad de tráfico importante y al firme se le quiere dotar de una alta calidad, habrá que pensar en esas soluciones para las capas inferiores, pero deberán proyectarse capas de rodadura de buena clase.

\section{SUELOS ESTABILIZADOS CON EMULSIÓN BITUMINOSA}

\section{Aspectos generales}

Las estabilizaciones con emulsión bituminosa han venido desarrollándose por dos diferentes procedimientos: en primer lugar, como estabilización in situ de zahorras naturales o suelos granulares y, en segundo, como fabricación de lo que se ha denominado grava-emulsión.

(c) Consejo Superior de Investigaciones Científicas Licencia Creative Commons 3.0 España (by-nc)

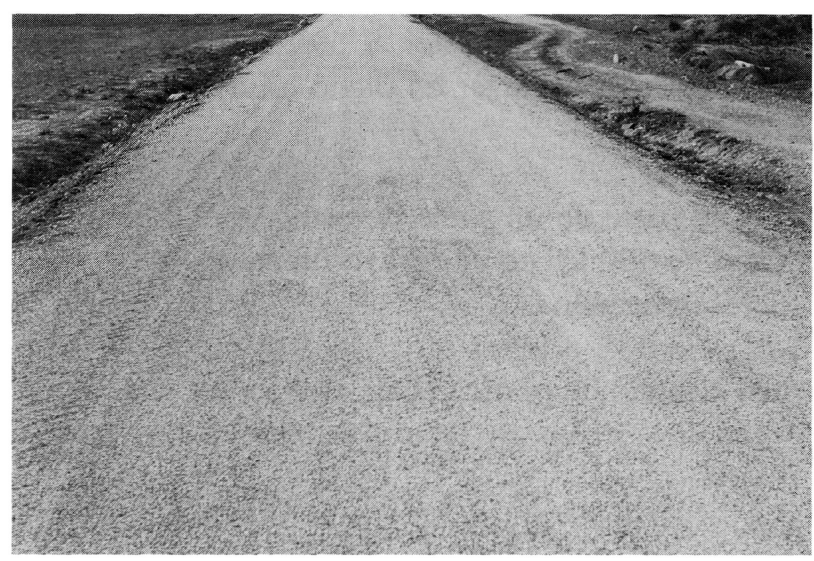

Fig. 13.- Aspecto con riego adicional de sellado a base de tratamiento superficial asfáltico.

Con el nombre de grava-emulsión es designado un tipo de mezcla bituminosa en frío constituida por áridos de granulometría continua, emulsión bituminosa y agua. Dicha mezcla se realiza en planta mezcladora de las mismas características que las utilizadas en la fabricación de cualquier otra mezcla asfáltica en frío.

El procedimiento de estabilización in situ básicamente es similar al anterior, aunque realizándose la mezcla sobre el propio camino y con la maquinaria habitual utilizada en la construcción de estas vías o de ciertas labores agrícolas (motoniveladoras, fresadoras, etc.). Lógicamente, esta última estabilización presenta como ventaja el ahorro en transporte de materiales (a la planta mezcladora y de la planta al camino) y como desventaja el realizar una peor homogeneización de la mezcla constituyente de la capa a construir.

Este método no supone ninguna novedad técnica, pues tiene su origen en las estabilizaciones de firmes por el procedimiento denominado "RETREAD PROCESS". Dicho método consiste en el reacondicionamiento de firmes con emulsión asfáltica. Se empleó, con mucha frecuencia, en la reparación de carreteras en Europa después de la Segunda Guerra Mundial. Principalmente se utilizó en firmes de macadam, que se escarificaban regando posteriormente con emulsión diluida y efectuando una nueva compactación.

Para estabilizaciones de suelos in situ con emulsión, se seguirán las recomendaciones del PG3 (Art. 511) de la Dirección General de Carreteras. Pafece lógico, pues, utilizar criterios similares a los utiłzzados en la grava-emulsión, como puede ser el ensayo de inmersión-compresión. Entonces, se ha de exigir una resis- 
tencia conservada superior al 50\%. En cuanto a las estabilidades a alcanzar en dicho ensayo han de ser las siguientes:

$1^{\circ}$ ) Si se parte de un suelo de categoría E2, un mínimo de $500 \mathrm{kp}$ en el ensayo en seco y de $350 \mathrm{kp}$ tras inmersión.

$2^{\circ}$ ) Si el suelo de partida es de categoría E3, un mínimo de $750 \mathrm{kp}$ en seco y de $500 \mathrm{kp}$ tras inmersión.

Normalmente en suelos estabilizados con emulsión asfáltica, se vienen utilizando porcentajes de betún residual entre el 2 y $5 \%$ sobre peso seco del suelo.

Las emulsiones empleadas normalmente son:

- Estabilización de suelos - EAL-2, ECL-2, EAM, ECM

- Grava-emulsión - EAL-2, ECL-2

Como ventajas de este procedimiento (aplicable tanto a la estabilización in situ como a la grava-emulsión) se pueden citar: impermeabilidad, resistencia a compresión y a deformaciones plásticas, aumento de capacidad portante flexibilidad versatilidad (frente a posible uso de materiales locales por desechables que parezcan, tipo de tráfico, climatología, etc.), excelente adherencia con otras capas bituminosas (se evita un riego de adherencia o imprimación), sencillez de fabricación y/o puesta en obra.

No obstante, es necesario señalar que este tipo de firme no constituye una buena solución en capas de rodadura, pues presenta poca resistencia a los esfuerzos tangenciales y por tanto debe evitarse (sin una capa protectora) en aquellos lugares en los que sean de prever frenadas, maniobras, etc. Es muy aconsejable, cuando se proyecte como capa última del firme, protegerla con un tratamiento superficial asfáltico (simple o doble) o una lechada bituminosa.

\section{Descripción del procedimiento de estabilización in situ}

En la ejecución de una estabilización de suelos es importante alcanzar la máxima disgregación del suelo. Para ello se pueden emplear gradas de disco, fresadoras, la propia motoniveladora, etc.

El procedimiento resumido en forma esquemática consiste en:

-Escarificado del suelo existente o extendido de la zahorra de aportación.
- Aireación del mismo o riego con agua hasta alcanzar la humedad adecuada, dependiendo del porcentaje de humedad que presente la zahorra o suelo a tratar. (Fig. 1.).

-Distribución de la emulsión bituminosa de la forma más regular posible. Se emplean las mismas cisternas que para un riego asfáltico. Normalmente se realiza en dos pases distintos para que haya una mejor penetración del ligante. (Figs. 2, 3 y 7 ).

-Mezcla con los equipos específicos (indicados anteriormente). Esta mezcla se realiza en tantas ocasiones como pases de distribución de emulsión asfáltica se realicen. (Figs. 4, 5, 6,8 y 9).

-Posteriormente se debe iniciar la compactación, para lo cual debe procurarse que el contenido de humedad sea óptimo. Si hay exceso es necesario un oreo previo y si hay defecto un riego con agua. (Figs. 10 y 11). Es conveniente utilizar rodillos vibratorios y de neumáticos conjuntamente.

-El tratamiento termina después de un período de veinticuatro horas de haberse alcanzado la densidad óptima de compactación (Fig. 12). No obstante es muy conveniente, si se va a utilizar como capa de rodadura, hacer un tratamiento final de sellado, ejecutando un tratamiento superficial asfáltico (simple o doble en función del tráfico previsto) (Fig. 13).

\section{ALTERNATIVA FRENTE A OTRAS ESTRUCTURAS DE FIRMES}

A la hora de proyectar la estructura de un firme concreto, habrá que analizar el tipo de vía de que se trate, las condiciones climáticas, el tráfico previsto, la disponibilidad de materiales adecuados, etc., para escoger de entre las distintas opciones existentes, la que en ese caso determinado presente las mayores ventajas técnicas y de calidad, en relación directa con el menor coste de inversión de las mismas.

No obstante y como muestra de la utilización de la emulsión bituminosa en estabilización de capas de firmes en caminos de baja intensidad de tráfico, como alternativa a otros métodos habitualmente empleados, se presentan algunas secciones estructurales equivalentes, por lo menos en cuanto a condiciones técnicas y de calidad se refiere (cuadro 5) 
Cuadro 5

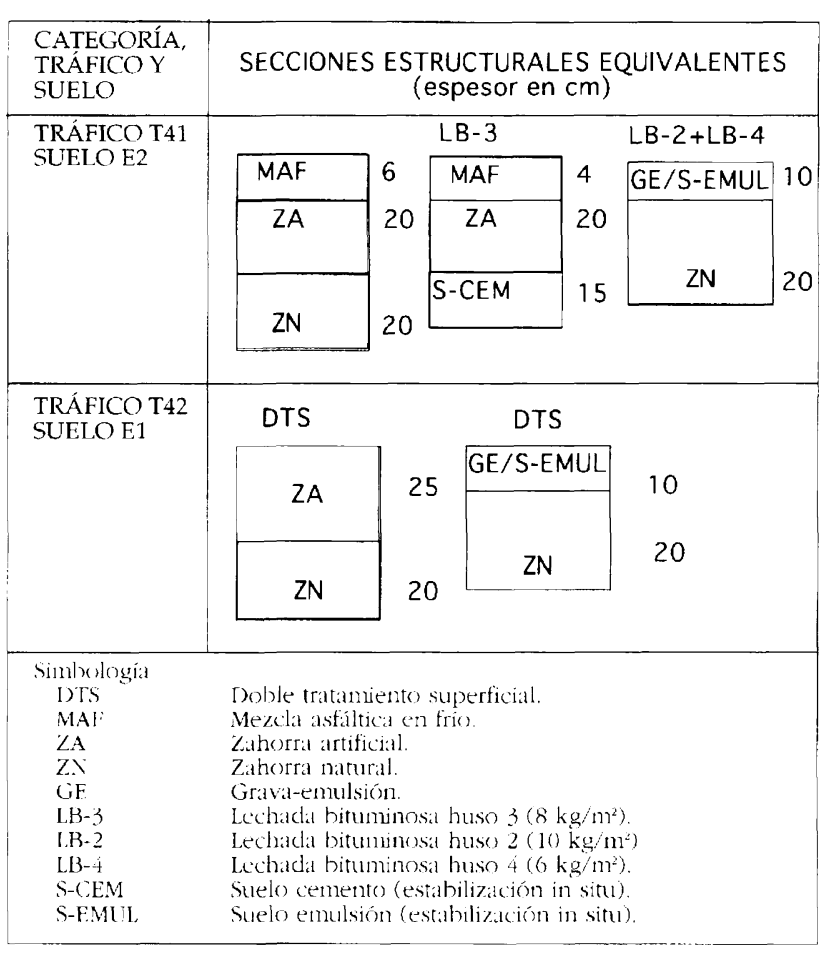

Lógicamente las soluciones anteriores hay que estudiarlas desde el punto de vista económico real de cada obra, ya que podrían variar mucho de unas a otras, en función de las características intrínsecas que presentase dicha obra. No obstante, si se establece una comparación utilizando unos precios medios, el coste total de la inversión, al menos en las secciones propuestas como ejemplo, generalmente es menor al de las soluciones que incluyen grava-emulsión o suelo-emulsión. No ya sólo referido al coste de construcción, sino también al de conservación de dicha inversión.

\section{CONCLUSIONES}

De lo expuesto se deduce que es factible realizar la construcción de caminos económicos en zonas con suelos de mala calidad, sin acudir a la estabilización natural, cuando ésta supone el deterioro del medio ambiente o un elevado costo.

La solución más conveniente consiste en emplear las propias explanaciones, previo tratamiento mejorados con materiales bituminosos, sobre las que se construirá el firme.

\section{BIBLIOGRAFÍA}

MOPU.- PLIEGO DE PRESCRIPCIONES TÉCNICAS GENERALES PARA OBRAS DE CARRETERAS Y PUENTES. PG 3 y 4.

MOPU.- MANUAL DE CONTROL DE FABRICACIÓN Y PUESTA EN OBRA DE MEZCLAS BITUMINOSAS 1978.

AYUSO MUÑOZ.- TRAZADO Y CÁLCULO DE CAMINOS RURALES.

ESCARIO Y OTROS.- CAMINOS.

IRYDA.- CAMINOS RURALES.- MANUALES TÉCNICOS.

JEUFFROY.- PROYECTO Y CONSTRUCCIÓN DE CARRETERAS.

ROAD RESEARCH LABORATORY.- MATERIALES BITUMINOSOS. 